\title{
Biolixiviación y su impacto en el rendimiento de aceite de semillas de Cucurbita foetidissima Kunth en dos métodos de extracción
}

\author{
Bioleaching and its impact on the yield of Cucurbita foetidissima Kunth \\ seed oil by two extraction methods
}

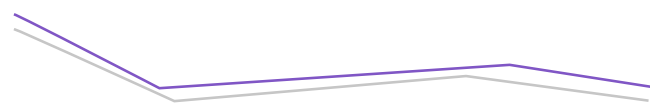

Francisco Hernández-Centeno*, Haydee Yajaira López-De la Peña*, César Guigón-López**, María Hernández-González*®

Hernández-Centeno, F., López-De la Peña, H. Y., Guigón-López, C., \& HernándezGonzález, M. (2018). Biolixiviación y su impacto en el rendimiento de aceite de semillas de Cucurbita foetidissima Kunth en dos métodos de extracción. Investigación y Ciencia de la Universidad Autónoma de Aguascalientes, 26(75), 13-19.
\end{abstract}

\section{RESUMEN}

La calabacilla loca (Cucurbita foetidissima Kunth) es una planta con amplio potencial de aprovechamiento. Se buscó determinar condiciones óptimas para extraer aceite de sus semillas, se les sometió a tratamiento previo de degradación de pared celular utilizando un hongo celulolítico para incrementar los rendimientos de aceite durante la extracción. El hongo Aspergillus niger, clasificado como GRAS, fue inducido en carboximetilcelulosa para promover producción de celulasas y después inocular un medio formulado con semilla de calabacilla loca como única fuente de carbono, más micronutrientes. Se utilizó prensado hidráulico y método Soxhlet para la extracción. Las mejores condiciones fueron: $\mathrm{pH} 7$ a $25^{\circ} \mathrm{C}$ por $96 \mathrm{~h}$, según la prueba de Tukey ( $p \leq$ 0.01 ). Con el método Soxhlet se obtuvo rendimiento de $34 \%$ para la muestra no tratada y $43.76 \%$ para la biolixiviada, y en el prensado fue de $24.44 \%$ para la

Palabras clave: calabacilla loca; aceite; biolixiviación; hongo; celulasas; rendimiento.

Keywords: buffalo gourd; oil; bioleaching; fungus; cellulases; performance.

Recibido: 11 de diciembre de 2017, aceptado: 14 de agosto de 2018

* Departamento de Ciencia y Tecnología de Alimentos, Universidad Autónoma Agraria Antonio Narro. Calzada Antonio Narro 1923 Buenavista, C. P. 25315, Saltillo, Coahuila, México. Correo electrónico: francisco.hdezc@gmail.com; yajaira.Ip@gmail.com; maryhg12@yahoo. com

** Centro de Investigación para los Recursos Naturales. Domicilio conocido. Antigua Normal Rural, Salaices, C. P. 33943, Villa López, Chihuahua, México. Correo electrónico: c guigon@hotmail.com

凶

Autor para correspondencia muestra no biolixiviada y $27.54 \%$ para muestra con tratamiento.

\section{ABSTRAC1}

The buffalo gourd (Cucurbita foetidissima Kunth) is a plant with a wide potential for use. We sought to determine optimal conditions to extract oil from their seeds, exposing them to previous treatment of cell wall degradation using a cellulolytic fungus to increase oil yields during extraction. The fungus Aspergillus niger, classified as GRAS, was induced in carboxymethylcellulose to promote production of cellulases and then inoculated a medium formulated with crazy gourd seed as the sole source of carbon, plus micronutrients. Hydraulic pressing and Soxhlet method were used for extraction. The best conditions were: $\mathrm{pH} 7$ at $25^{\circ} \mathrm{C}$ for $96 \mathrm{~h}$, according to the Tukey test ( $p \leq 0.01$ ). With the Soxhlet method, a yield of $34 \%$ was obtained for the untreated sample and $43.76 \%$ for the biolix spill, and in the press was $24.44 \%$ for the non-biowashed sample and $27.54 \%$ for the sample with treatment.

\section{INTRODUCCIÓN}

El uso de aceites comestibles se ha vuelto indispensable en la vida cotidiana en la preparación de alimentos, los cuales imparten características importantes relacionadas con su degustación, como el sabor y textura, tanto en alimentos naturales como preparados (Murcia, 2010), por este motivo tienen gran demanda en el mercado. Los aceites de semillas constituyen $70 \%$ de la producción mundial de aceites (Grasso, 2013) y en México su producción 
IIVESTIGACIÓn Y CIERCIA DE LA UNIVERSIDAD AUTÓNOMA DE RGUASCALIERTES requiere de la importación cada vez mayor de oleaginosas (SAGARPA, 2015).

La calabacilla loca (C. foetidissima Kunth) (figura 1) representa una alternativa para la producción de aceite pues sus semillas son ricas en él, oscila entre 25 y $42.8 \%$ de su peso, se compone principalmente de ácidos grasos no saturados como el ácido linoleico (Granados Sánchez y López Ríos, 1999; Stevenson et al., 2007), similar al contenido de aceite en semillas de otras cucurbitáceas (Seymen, Uslu, Türkmen, Al Juhaimi, \& Özcan, 2016). Además, es una especie silvestre y perenne que crece en forma natural en zonas áridas y semiáridas de México (Lira Saade, Eguiarte Fruns, \& Montes Hernández, 2009).

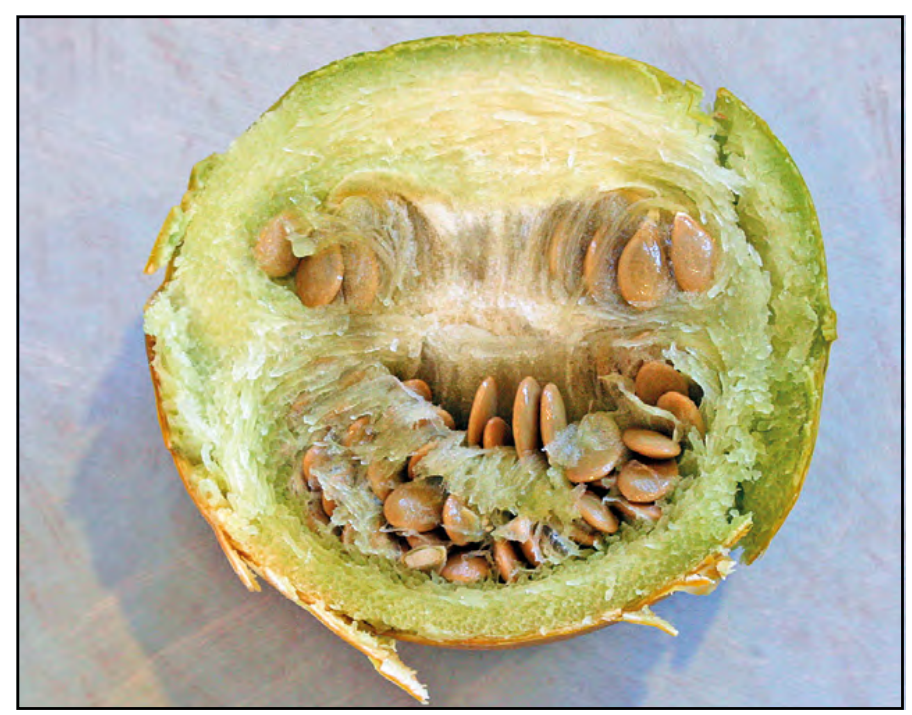

Figura 1. Fruto y semillas de calabacilla loca (C. foetidissima Kunth).

Fotografía tomada de Kleinman (2009).

La extracción de aceites se realiza mediante métodos físicos y químicos (Da Porto, Porretto, \& Decorti, 2013; Ixtaina et al., 2011); sin embargo, la sociedad actual exige alimentos con mayor calidad. Dentro de estas exigencias, una de las más importantes es la elaboración de alimentos por procesos físicos, donde no intervengan procesos químicos que puedan afectar la salud del consumidor (Monteiro, Bertazzy Levy, Moreira Claro, Ribeira de Castro, \& Cannon, 2010). Uno de estos campos de investigación involucra la biotecnología aplicada en alimentos (Kuhad, Gupta, \& Singh, 2011), que se ha desarrollado ampliamente en los últimos años con la finalidad de optimizar los procesos de transformación de los mismos (Latif \& Anwar, 2011; Sabiha-Hanim, Noor, \& Rosma, 2011).
El objetivo de este estudio fue comprobar que la tecnología de biolixiviación fúngica es una alternativa efectiva para aumentar el rendimiento final en la extracción de aceite de semillas de calabacilla loca (C. foetidissima Kunth) sin importar el método de extracción y con impacto ambiental menor al de los procesos de obtención convencionales.

\section{MATERIALES Y MÉTODOS}

Para el proceso de biolixiviación se utilizó un hongo aislado a partir de una muestra de agar contaminado. En la inducción del hongo se empleó caldo Czapeck Dox enriquecido con sacarosa como única fuente de carbono, además de micronutrientes y se incubó entre 7 y 9 días a $25^{\circ} \mathrm{C}$. Posteriormente se sustituyó poco a poco la sacarosa por concentraciones crecientes de carboximetilcelulosa (CMC) hasta ser la única fuente de carbono e inducir al hongo a producir celulasas, según lo referido por Tirado Gallegos (2005), con modificaciones.

Para la caracterización se realizó microcultivo del hongo con la finalidad de determinar su tipo y factibilidad de uso. Se caracterizó de acuerdo con los lineamientos de Barnett y Hunter (1998). La subespecie se determinó de acuerdo con las claves de identificación de Aspergillus sp. según Raper y Fennell (1965).

Se establecieron las condiciones óptimas de degradación de la pared celular, para lo cual se monitoreó el contenido de azúcares totales. Esta etapa se llevó a cabo en medio acuoso con valores de $\mathrm{pH}$ de 3,5 y 7 , se sustituyó la sacarosa de manera gradual por semilla de calabacilla molida a tamaño $\leq 1 \mathrm{~mm}$ como única fuente de carbono, además de micronutrientes. Se preparó un inóculo con $2 \times 10^{6}$ esporas/l, al cual se adicionó $1 \mathrm{ml} / 100 \mathrm{ml}$ de medio acuoso, con el sistema en agitación constante. El conteo de esporas se realizó de acuerdo con lo reportado por Tirado Gallegos (2005). Las muestras se mantuvieron en baño María a $25^{\circ} \mathrm{C}$ durante 168 h. Durante el tiempo de incubación se evaluó cada $24 \mathrm{~h}$ el contenido de azúcares totales (DuBois, Gilles, Hamilton, Rebers, \& Smith, 1956) y reductores (Miller, 1959). Se retiró el micelio del material ya biolixiviado, se filtró en tela de lino y se secó en estufa Robertshaw durante $24 \mathrm{~h}$ a $55^{\circ} \mathrm{C}$.

Para la obtención de la semilla predigerida se siguió la metodología que se describió en el 


\section{InVESTIGACIÓn Y CIERCIA DE LA UחIVERSIDAD AUTÓnOMA DE RGUASCALIEחTES}

punto anterior, mediante las condiciones óptimas derivadas del análisis durante este mismo estudio: $\mathrm{pH} 7$ y $96 \mathrm{~h}$ de contacto, a fin de obtener suficiente material para las etapas posteriores del experimento. En cuanto a la evaluación del efecto del tratamiento biolixiviante en la eficiencia de extracción de aceite se utilizó muestra biolixiviada y sin biolixiviar, esta última como testigo de referencia. La primera tecnología de extracción fue el método Soxhlet (Horwitz \& Latimer, 2010) con modificaciones: se utilizaron $250 \mathrm{ml}$ de hexano por cada $2 \mathrm{~g}$ de muestra, tanto para el testigo como para el tratamiento; el proceso se llevó a $60^{\circ} \mathrm{C}$ en diferentes tiempos (6, 8, 10 y 12 h). Para la segunda tecnología se utilizó una prensa hidráulica, en la cual se colocaron muestras de $2 \mathrm{~g}$ a una presión de 30,000 psi por $3 \mathrm{~s}$ en tres ocasiones consecutivas.

Se obtuvo el espectro infrarrojo del aceite obtenido utilizando un espectrofotómetro FTIR modelo Nicolet 550, a fin de descartar la presencia de componentes ajenos. El diseño experimental fue al azar, los promedios de rendimiento obtenido se analizaron de acuerdo con la prueba de comparación de medias de Tukey $(p \leq 0.01)$, con el software estadístico JMP 5.0.1.

\section{RESULTADOS}

La figura 2 muestra el crecimiento del hongo en medio con concentración de $100 \%$ de CMC. La caracterización macroscópica y microscópica hizo posible la identificación de la cepa como Aspegillus niger (figura 3).

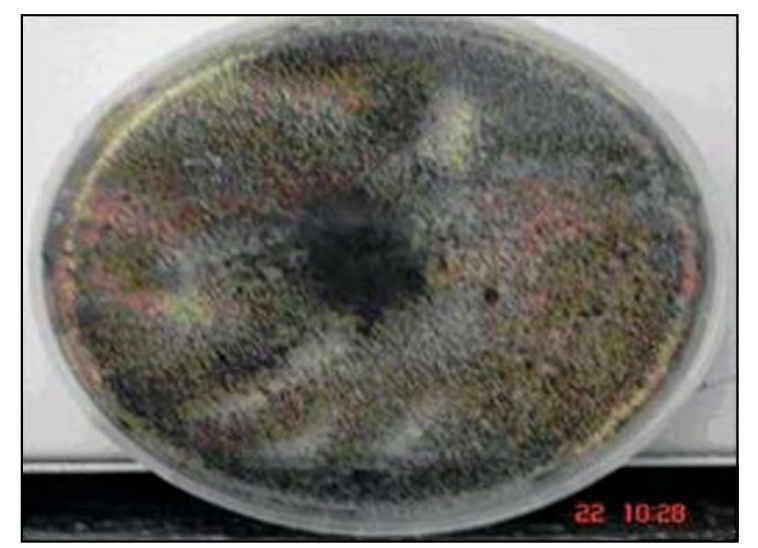

Figura 2. Cultivo celular del hongo Aspergillus sp. en un medio con concentración de $100 \%$ de carboximetilcelulosa (CMC).

Fotografía de los autores.

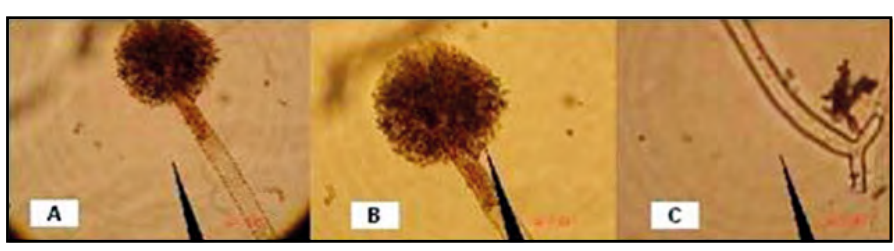

Figura 3. Morfología de Aspergillus sp. A y B) Cabezas conidiales globosas y esporas en cadena sobre conidióforos. C) Célula pie. Fotografías de los autores.

El proceso de inducción del microorganismo se llevó a cabo de forma exitosa: la formación de azúcares totales y su asimilación por el hongo en uso se pueden observar claramente en la figura 4, donde se aprecian los ciclos de liberación y asimilación de estos nutrientes.

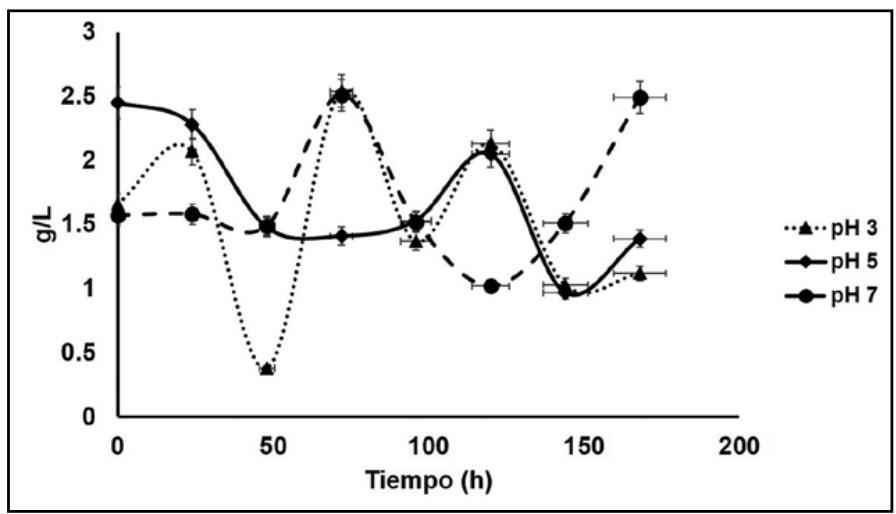

Figura 4. Concentración de azúcares totales a pH 3,5 y 7 a $25^{\circ} \mathrm{C}$ por $168 \mathrm{~h}$.

Elaboración propia.

En el sistema de biolixiviación fúngica, con las semillas molidas como única fuente de carbono, la actividad de $A$. niger mostró un comportamiento diferente entre formación y asimilación de azúcares reductores con los valores de $\mathrm{pH}$ durante el mismo tiempo de evaluación (figura 5).

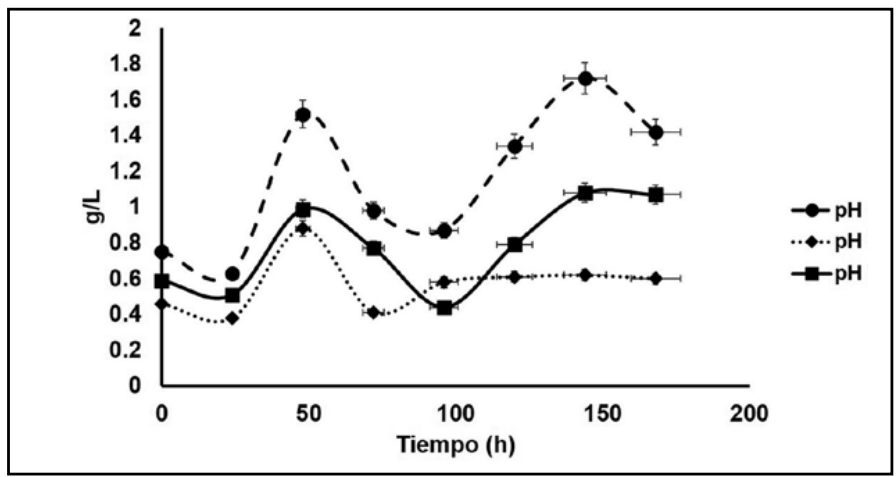

Figura 5. Concentración de azúcares reductores a pH 3, 5 y 7 a $25^{\circ} \mathrm{C}$ por $168 \mathrm{~h}$.

Elaboración propia. 


\section{INVESTIGAGIÓก Y CIECEIA DE LA UחIVERSIDAD AUTÓกOMA DE AGUASCALIERTES}

Se apreció a simple vista un cambio de tonalidad de color en el material tratado a consecuencia del tratamiento de prueba, mediante el uso para el sistema de las condiciones que demostraron ser más efectivas en cuanto a liberación de azúcares reductores. Esto también influyó en el color del aceite crudo, aunque esta característica no fue evaluada.

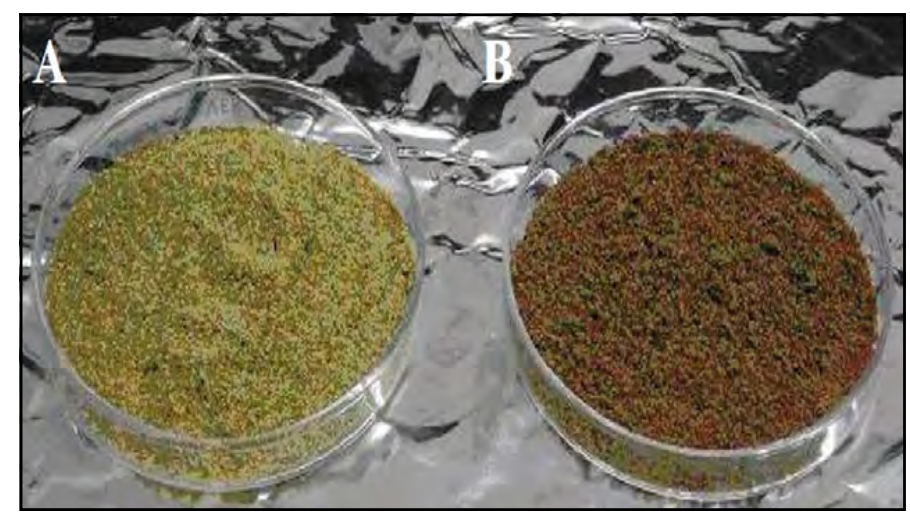

Figura 6. Semilla molida de Cucurbita foetidissima Kunth no tratada (A) y biolixiviada con A. niger por $48 \mathrm{~h}, 25^{\circ} \mathrm{C}$ y $\mathrm{pH} 7$ (B). Fotografía de los autores.

Para la extracción de aceite previa a la biolixiviación, en la figura 7 se apreció que el mejor tiempo de extracción es a las 12 h; sin embargo, la diferencia entre las 12,4 y $6 \mathrm{~h}$ no fue significativa ( $p$ $\leq 0.01$ ) en cuanto a la cantidad de aceite obtenido, por lo que se consideró como mejor el de $4 \mathrm{~h}$.

El rendimiento de aceite del material tratado obtenido a través de ambos métodos de extracción

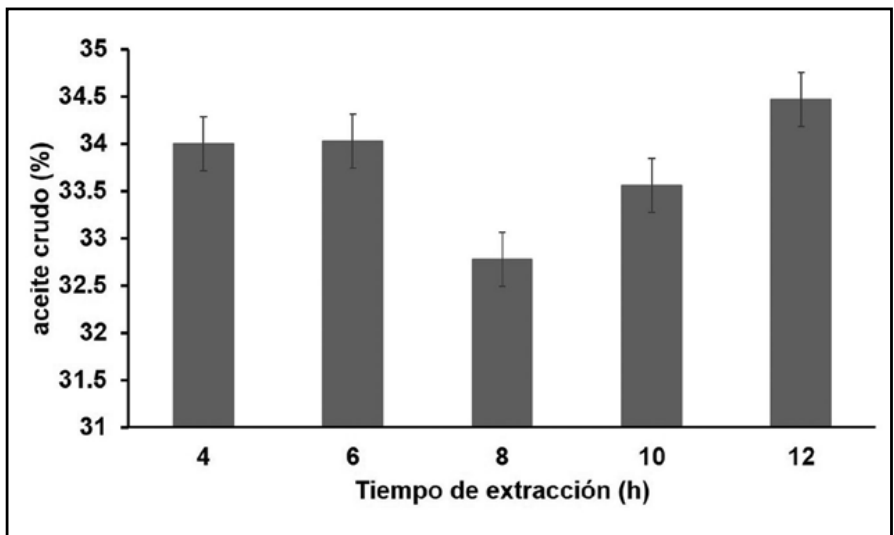

Figura 7. Porcentaje de aceite obtenido de semillas de C. foetidissima Kunth, molidas, sin tratamiento, utilizando el método Soxhlet.

Elaboración propia. se comparó con el testigo sin tratar y se observó una menor cantidad de aceite para el método de prensado (figura 8) con respecto al obtenido por método Soxhlet (figura 9).

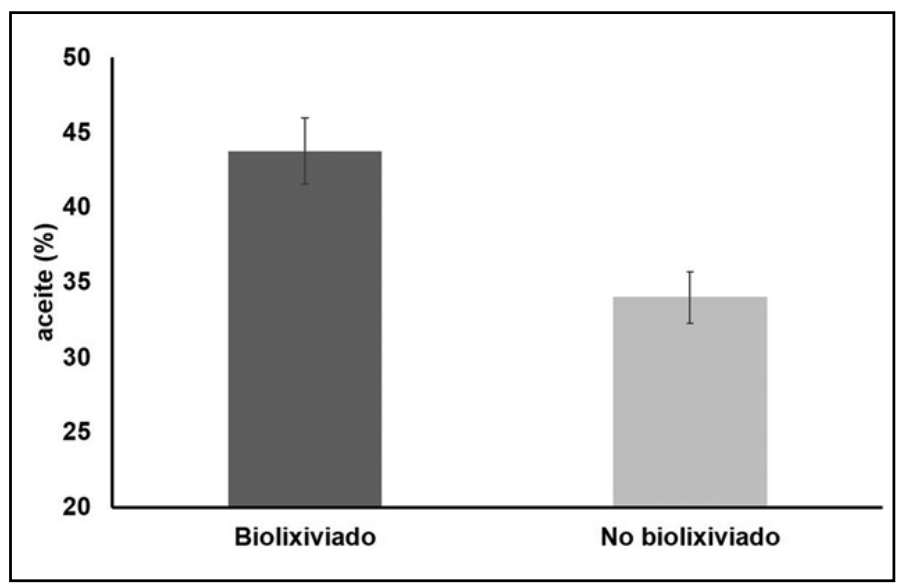

Figura 8. Porcentajes de aceite obtenido por método Soxhlet (4 h) de semillas molidas de C. foetidissima Kunth biolixiviadas y $\sin$ biolixiviar.

Elaboración propia.

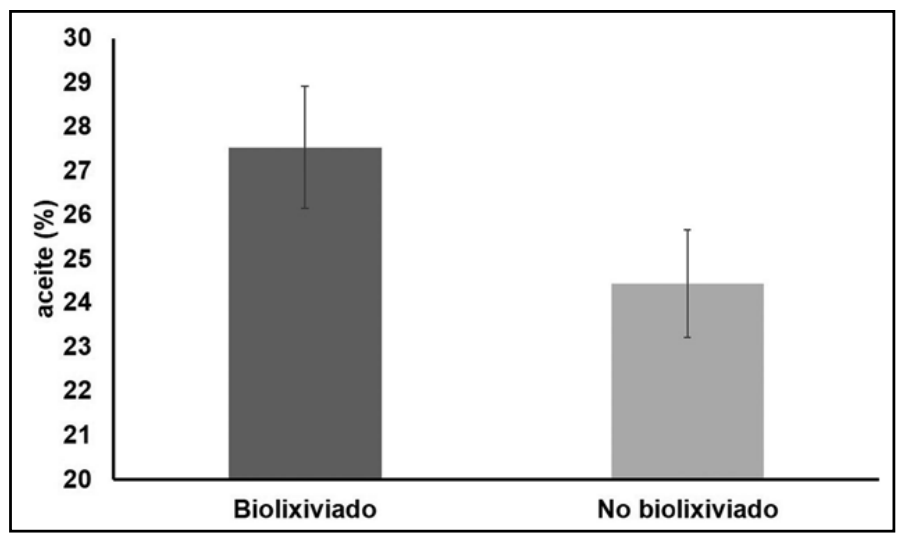

Figura 9. Porcentajes de aceite obtenido por método de prensado de semillas molidas de $C$. foetidissima Kunth biolixiviadas y sin biolixiviar.

Elaboración propia.

Para el análisis FTIR, el espectro obtenido del análisis de la muestra de aceite crudo de semilla de calabacilla loca se muestra en la figura 10.

\section{DISCUSIÓN}

La cepa de hongo analizada se clasificó como Aspergillus sp. y se caracterizó con el acrónimo GRAS (generally recognized as secure). La observación microscópica permitió una tipificación más precisa. El color presentado en las colonias de Aspergillus 


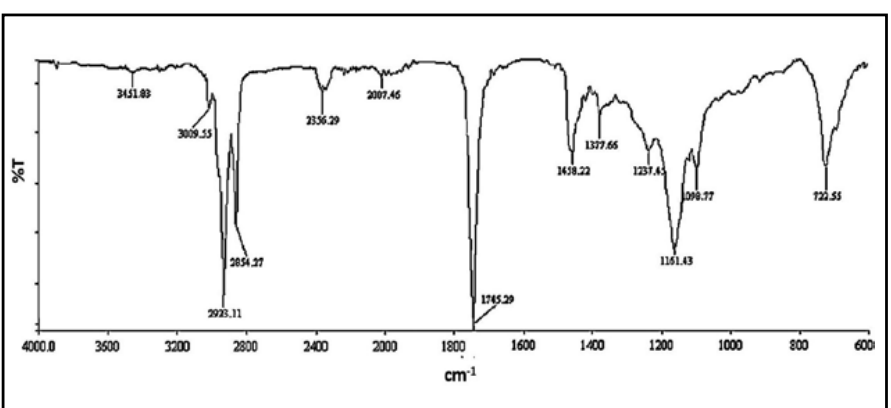

Figura 10. Espectro IR del aceite crudo extraído de semilla de calabacilla loca (C. foetidissima Kunth).

Elaboración propia.

fue negruzco, lo cual permitió identificar la especie como niger. De acuerdo con Raper y Fennell (1965), A. niger presenta cabezuelas color negro o café oscuro y conidióforos lisos e incoloros o pigmentados debajo de la vesícula (figura 3).

El proceso de desdoblamiento y asimilación de los azúcares se debió al metabolismo del hongo inoculado, que da lugar a la hidrólisis de los polímeros de celulosa presentes en la pared celular de las semillas en estudio. Deacon (1988) menciona que durante la actividad del hongo se absorben los nutrientes simples y solubles que se liberan al medio como resultado de la degradación de polímeros complejos con enzimas extracelulares, por lo que la síntesis del complejo celulasa es reprimida por altos niveles de glucosa u otro azúcar fácilmente metabolizable.

Tomando en cuenta los resultados mostrados en la figura 4 se determinó que a $\mathrm{pH} 7$ y $25^{\circ} \mathrm{C}$ el hongo tiene mejor desarrollo; su comportamiento en el medio bajo esas condiciones tiende a ser equilibrado a partir de las $72 \mathrm{~h}$ con un intervalo de $48 \mathrm{~h}$ entre la asimilación y la formación de azúcares totales; por tanto, su actividad celulolítica es más eficiente, al degradar de manera constante la pared celular y permite mayor liberación de aceite en el tratamiento de extracción.

Las condiciones que permitieron mejores resultados en la liberación de azúcares totales mostraron también los mejores resultados para los reductores, solo que a partir de las $48 \mathrm{~h}$ y mostraron un comportamiento constante hasta las $96 \mathrm{~h}$, lo que permite sugerir que este tiempo de proceso fue el más adecuado para realizar la biolixiviación en función del costo-beneficio del tratamiento, ya que a pesar de que después se alcanzan mayores niveles de azú- cares, no se observaron aumentos significativos en rendimiento de aceite en tiempos posteriores al indicado. El mayor rendimiento de aceite $(p \leq 0.01)$ se obtuvo con el tratamiento a pH 7.

En la semilla tratada con biolixiviación la degradación de la pared celular permitió que el aceite quedara libre. Esto concuerda con los resultados obtenidos en estudios similares (Hernández, Monroy, Hernández, \& De la Garza, 2008; Rosenthal, Pyle, Nirvanjan, Gilmour, \& Trinca, 2001), donde los rendimientos de producto también aumentaron por un efecto similar. El mejor tiempo de extracción de aceite por el método Soxhlet fue de $4 \mathrm{~h}$, si se considera que no hubo diferencia significativa $(p \leq 0.01)$ con los tratamientos más prolongados, los cuales presentaron la desventaja de arrastrar otros componentes que no son aceite, principalmente pigmentos, y con el fin de aprovechar al máximo los recursos. La disminución en el rendimiento de aceite obtenido a las $8 \mathrm{~h}$ se debe a que la micela que se formó en el solvente durante el proceso de extracción llegó casi a la saturación, de acuerdo con Marjury Delgado et al. (2010) la concentración del aceite en función del tiempo disminuye hasta hacerse asíntota por un efecto de contracorriente en el cual el producto avanza en sentido contrario al del solvente.

En cuanto a la comparación de rendimientos entre semilla biolixiviada y sin biolixiviar, al aplicar celulasas se obtuvieron mayores rendimientos ( $p \leq 0.01$ ) en la extracción con solvente (figura 7), lo que concuerda con resultados obtenidos por Tano-Debrah y Ohta $(1994,1995)$, ya que reportaron también mayor rendimiento de producto final al utilizar un sistema de predigestión enzimática.

Para el método de extracción por prensado los resultados también presentaron diferencia ( $p \leq 0.01$ ) entre los rendimientos obtenidos de muestra biolixiviada y de muestra testigo sin biolixiviar (figura 8), ya que de la muestra tratada se pudo obtener $9.76 \%$ más de aceite con respecto a la muestra testigo, lo cual coincide con lo encontrado por Bouvier y Entressangles (1992) y Cheah, Agustin y Ooi (1990) para pretratamiento enzimático en la obtención de aceite de palma, aunque es significativamente menor al incremento en rendimiento que obtuvieron Guerra y Zúñiga (2003) para la extracción de aceite de pepita de uva tratada enzimáticamente; sin embargo, es suficiente para establecer que el uso de hongos celulolíticos es efectivo para el tratamiento de biolixiviación y obtener mejores rendimientos de producto final. 
IIVESTIGACIÓn Y CIERCIA DE LA UNIVERSIDAD AUTÓNOMA DE RGUASCALIERTES
La extracción mediante solventes rindió un mayor porcentaje de aceite en comparación con el prensado, lo cual es congruente con un estudio similar sin pretratamiento llevado a cabo por BrossardGonzález, Ferrari, Pighinelli y Park (2010); sin embargo, el hexano utilizado en la primera metodología es un material tóxico que al exponerse por tiempos prolongados a temperaturas elevadas tiende a descomponerse generando compuestos nocivos para el medio ambiente, así como para la salud de los consumidores (Finlayson-Pitts \& Pitts, 1993; Mustakas, 1980). Por lo anterior, es de esperarse que el método de prensado provoca un menor impacto ambiental al estar ausente cualquier agente químico ajeno al material en proceso, aunado a que con un proceso previo de biolixiviación de las semillas molidas se obtiene un mayor rendimiento durante la extracción.

Los resultados del FTIR demostraron una estructura correspondiente a los ácidos grasos linoleico, oleico y palmítico, típica de la estructura de los aceites vegetales vírgenes que se ha reportado en otros estudios, como los de Che Man y Setiowaty (1999) y Hernández y Zacconi (2009).

\section{CONCLUSIONES}

La inducción del hongo para la producción de celulasas se logró utilizando carboximetilcelulosa. Las condiciones óptimas del tratamiento de biolixiviación fueron $\mathrm{pH} 7.25^{\circ} \mathrm{C}$ y $96 \mathrm{~h}$ de contacto. El rendimiento de aceite por el método de extracción Soxhlet para las semillas sin biolixiviar fue mayor que con el método de prensado para este mismo material. Lo mismo ocurrió con las semillas biolixiviadas, con mayor rendimiento en ambos métodos; sin embargo, la obtención por prensado fue mejor por su conveniencia ambiental y por ser menos exigente en cuanto a recursos necesarios para la obtención del aceite.

\section{REFERENCIAS}

- Barnett, H. L., \& Hunter, B. B. (1998). Ilustrated general of imperfect fungi ( $4^{\text {th. }}$ ed.). St. Paul, MN, US: APS Press.

- Bouvier, F., \& Entressangles, B. (1992). Utilisation de cellulases et pectinases dans le procédé d'extraction de l'huile de palme. Revue Francaise des Corps Gras, 39(9), 243-314. Recuperado de http://bibliotheque.bordeaux.fr/in/details.xhtmle id= mgroup\%3Ap+unimarcbu_32860\&jscheck=1

- Brossard-González, C. O., Ferrari, R. A., Pighinelli, A. L., \& Park, K. J. (2010). Evaluación preliminar del etanol anhidro como solvente en la extracción de aceite de semillas de jatrofa (Jatropha curcas L.). Grasas y aceites, 61 (3), 295-302. doi: 10.3989/gya.087209

- Che Man, Y. B., \& Setiowaty, G. (1999). Multivariate calibration of Fourier transform infrared spectra in determining iodine value of palm oil products. Food Chemistry, 67(2), 193-198. doi: 10.1016/S0308-8146(99)00102-8

- Cheah, S. C., Augustin, M. A., \& Ooi, L. C. L. (1990). Enzymic extraction of palm oil. PORIM Bulletin, 20, 30-36. Recuperado de https://www.cabdirect.org/cabdirect/abstract/19920310811

- Da Porto, C., Porretto, E., \& Decorti, D. (2013). Comparison of ultrasound-assisted extraction with conventional extraction methods of oil and polyphenols from grape (Vitis vinifera L.) seeds. Ultrasonics Sonochemistry, 20(4), 1076-1080. doi: 10.1016/j.ultsonch.2012.12.002

- Deacon, J. W. (1988). Introducción a la micología moderna. México, D. F.: Limusa.
- DuBois, M., Gilles, K. A., Hamilton, J. K., Rebers, P. A., \& Smith, F. (1956). Colorimetric method for determination of sugars and related substances. Analytical Chemistry, 28(3), 350-356. doi: $10.1021 / a c 60111 \mathrm{a} 017$

- Finlayson-Pitts, B. J., \& Pitts, J. N. (1993). Volatile organic compounds: Ozone formation, alternative fuels, and toxics. Chemistry and Industry, 20, 796-800. Recuperado de https:// www.osti.gov/etdeweb/biblio/5703621

- Granados Sánchez, D., \& López Ríos, G. F. (1999). Un recurso forestal de zonas áridas: Calabacilla loca (Cucurbita foetidissima H.B.K.). Revista Chapingo. Serie ciencias forestales y del ambiente, 5(1), 35-40.

- Grasso, F. V. (2013). Diseño del proceso: Pretratamiento enzimático para extracción de aceites vegetales en un extractor de columna (Tesis doctoral). Recuperada de http:// sedici.unlp.edu.ar/handle/10915/26889

- Guerra, E. G., \& Zúñiga, M. E. (2003). Tratamiento enzimático en la extracción de aceite de pipa de uva, Vitis vinifera, por prensado en frío. Grasas y aceites, 54(1), 53-57. Recuperado de https://dialnet.unirioja.es/servlet/articulo? codigo $=625793$

- Hernández, M., Monroy, A., Hernández, F., \& De la Garza, H. (2008). Obtención de aceite de calabacilla loca (Cucurbita foetidissima) aplicando biolixiviación para mejorar su rendimiento. Libro de resúmenes del XIII Congreso Anual en Ciencia y Tecnología de los Alimentos. Madrid, España: Universidad Complutense. Recuperado de https:// es.slideshare.net/Alcyta/libro-ponencias-cytalia-xiii 


\section{IIVESTIGAGIÓn Y CUERGIA DE LA UחIVERSIDAD AUTÓnOMH DE RGUASCHLIETTES}

- Hernández, S. A., \& Zacconi, F. C. M. (2009). Aceite de almendras dulces: Extracción, caracterización y aplicación. Química Nova, 32 (5), 1342-1345. Recuperado de http://dx.doi. org/10.1590/S0100-40422009000500044

- Horwitz, W., \& Latimer, G. (Eds.). (2010). Official Methods of Analysis of AOAC International (18 $8^{\text {th }}$ ed., 3rd. rev.). Arlington, TX, US: AOAC International.

- Ixtaina, V. Y., Martínez, M. L., Spotorno, V., Mateo, C. M., Maestri, D. M., Diehl, B. W. K., ..., Tomás, M. C. (2011). Characterization of chia seed oils obtained by pressing and solvent extraction. Journal of Food Composition and Analysis, 24 (2), 166-174. doi: 10.1016/j.jfca.2010.08.006

- Kleinman, R. (2009). Cucurbita foetidissima, gourd cut in half [Fotografía]. Recuperada de https://wnmu.edu/academic/ nspages/gilaflora/cucurbita_foetidissima.html

- Kuhad, R. C., Gupta, R., \& Singh, A. (2011). Microbial cellulases and their industrial applications. Enzyme Research, 2011 ID 280696. Recuperado de http://dx.doi.org/10.4061/201 1/280696

- Latif, S., \& Anwar, F. (2011). Aqueous enzymatic sesame oil and protein extraction. Food Chemistry, 125(2), 679-684. doi: 10.1016/j.foodchem.2010.09.064

- Lira Saade, R., Eguiarte Fruns, L., \& Montes Hernández, S. (2009). Proyecto recopilación y análisis de la información existente de las especies de los géneros Cucurbita y Sechium que crecen y/o se cultivan en México (Informe final). México, D. F.: Universidad Nacional Autónoma de México-Instituto Nacional de Investigaciones Agrícolas, Forestales y Pecuarias. Recuperado de http://www.biodiversidad.gob.mx/genes/ centrosOrigen/Cucurbita\%20y\%20Sechium/Informe_Final/ Informe\%20final\%20Cucurbita\%20y\%20Sechium.pdf

- Marjury Delgado, R., Modroño-Alonso, M. F., Mujica F., V. C., Ramírez S., M., Rodríguez, M. C., Yamal T., E., \& Velásquez N., I. (2010). Evaluación del proceso de extracción de aceite de la semilla de Merey (Anacardium occidentale L.) a escala piloto en el Laboratorio de Ingeniería Química de la Universidad de Carabobo. Revista Técnica de la Facultad de Ingeniería Universidad del Zulia, 33(1), 39-97.

- Miller, G. L. (1959). Use of dinitrosalicylic acid reagent for determination of reducing sugar. Analytical Chemistry, 31 (3), 426-428. doi: 10.1021/ac60147a030

- Monteiro, C. A., Bertazzi Levy, R., Moreira Claro, R., Ribeira de Castro, I. R., \& Cannon, G. (2010). Increasing consumption of ultra-processed foods and likely impact on human health: Evidence from Brazil. Public Health Nutrition, 14(1), 5-13. doi: $10.1017 /$ S1368980010003241

- Murcia, J. L. (2010). Aceites de semillas: Palma, colza, soja y girasol lideran la producción y el consumo mundial. Distribución y Consumo, 111, 65-70. Recuperado de http://www.mercasa. es/files/multimedios/pag_065-070_Murcia.pdf

- Mustakas, G. C. (1980). Recovery of oil from soybeans. En D. R. Erikson, E. H. Pryde, O. L. Brekke,T. L. Mounts, \& R. A. Falb (Eds.), Handbook of soy oil processing and utilization. St. Louis, US: American Soybean Association-American Oil Chemist's Society.

- Raper, K. B., \& Fennell, D. I. (1965). The genus Aspergillus. Baltimore, US: Williams and Wilkins.

- Rosenthal, A., Pyle, D. L.; Nirvanjan, K., Gilmour, S., \& Trinca, L. (2001). Combined effect of operational variables and enzyme activity on aqueous enzymatic extraction of oil and protein from soybean. Enzyme and Microbial Technology, 28(6), 499509. doi: 10.1016/S0141-0229(00)00351-3

- Sabiha-Hanim, S., Noor, M. A. M., \& Rosma, A. (2011). Effect of autohydrolysis and enzymatic treatment on oil palm (Elaeis guineensis Jacq.) frond fibres for xylose and xylooligosaccharides production. Bioresource Technology, 102(2), 1234-1239. doi: 10.1016/j.biortech.2010.08.017

- Secretaría de Agricultura, Desarrollo Rural, Pesca y Alimentación (SAGARPA). (2015). Aumenta México 6\% producción de granos y oleaginosas en 2014. Comunicado de prensa. Culiacán, Sinaloa, México, 18 de febrero de 2015. Recuperado de http://www. sagarpa.gob.mx/saladeprensa/2012/Paginas/2015B120.aspx

- Seymen, M., Uslu, N., Türkmen, Ö., Al Juhaimi, F., \& Özcan, M. M. (2016). Chemical compositions and mineral contents of some hull-less pumpkin seed and oils. Journal of the American Oil Chemists' Society, 93(8), 1095-1099. Recuperado de https://link. springer.com/article/10.1007/s1 1746-016-2850-5

- Stevenson, D. G., Eller, F. J., Wang, L., Jane, J. L., Wang, T., \& Inglett, G. E. (2007). Oil and tocopherol content and composition of pumpkin seed oil in 12 cultivars. Journal of Agricultural and Food Chemistry, 55(10), 4005-4013. doi: 10.1021/jf0706979

- Tano-Debrah, K., \& Ohta, Y. (1994). Enzyme-assisted aqueous extraction of fat from kernels of shea tree, Butyrospermum parkii. Journal of the American Oil Chemists' Society, 71 (9), 979983. Recuperado de https://link.springer.com/article/10.1007/ BF02542265

fat: A rural approach. Journal of the American Oil Chemists' Society, 72(2), 251-256. Recuperado de https://link.springer. com/article/10.1007/BF02638908

- Tirado Gallegos, J.M. (2005).Obtención del colorante delasemilla deachiote (Bixa orellana) utilizandomicroorganismoscelulolíticos (Tesis de licenciatura). Recuperada de http://repositorio.uaaan. mx:8080/xmlui/bitstream/handle/123456789/343/T15084\%20 TIRADO\%20GALLEGOS\%2C\%20JUAN\%20MANUEL\%20\%20TESIS. pdf? sequence $=1$ 